\title{
EFICIÊNCIA DO ATENDIMENTO ASSISTENCIAL NOS HOSPITAIS UNIVERSITÁRIOS
}

\author{
Samuel Cavalcante Mota ${ }^{1}$ \\ Antonio Rafael Valério de Oliveira ${ }^{2}$ \\ Alessandra Carvalho de Vasconcelos ${ }^{3}$
}

- Artigo recebido em: 15/07/2021 •- Artigo aceito em: 07/09/2021 -* Segunda versão aceita em: 29/10/2021

\section{RESUMO}

O aumento dos gastos com saúde, afeta mais os países em desenvolvimento, sobretudo em função do envelhecimento populacional, da inflação dos custos médicos e da polarização econômica e social. Um dos maiores desafios para o gestor público, em especial de saúde, consiste em buscar ganhos de eficiência, já que os recursos são escassos. Referente aos hospitais universitários, a complexidade do atendimento assistencial é agravada pelo acréscimo das atividades de ensino e pesquisa. A criação da Empresa Brasileira de Serviços Hospitalares (EBSERH) em 2011 implementou a gestão empresarial, onde se acentua a pressão por eficiência. Nesse contexto, o estudo avalia a eficiência do atendimento assistencial nos hospitais universitários federais geridos pela EBSERH. Foi procedida a Análise por Envoltória de Dados (DEA), Modelo BCC (BankerCharnes-Cooper), com orientação para outputs, para o cálculo da eficiência dos 40 hospitais que mantêm contrato com a EBSERH. Foram aplicados correlação e testes de médias sobre os dados de 2018. Os resultados indicam que 10 hospitais são eficientes, sendo seis localizados na Região Nordeste. Dentre os cinco hospitais com os mais baixos níveis de eficiência, incluem-se os dois do Rio de Janeiro. A taxa de mortalidade foi a variável que apresentou o maior potencial de melhoria, requerendo mais atenção dos gestores. Não foi identificada

\footnotetext{
1 Mestre em Administração e Controladoria pela Universidade Federal do Ceará (UFC), Contador da Universidade Federal do Ceará (UFC). Endereço: Av. da Universidade, 2853, Coordenadoria de Contabilidade e Finanças, Pró-Reitoria de Planejamento e Administração, Bairro Benfica, Fortaleza/CE, Brasil, CEP: 60020-181. Telefone: (85) 3366-7934. E-mail:
} samuelcmota@hotmail.com.

https://orcid.org/0000-0001-9197-1467

2 Mestre em Administração e Controladoria pela Universidade Federal do Ceará (UFC), Contador da Universidade Federal do Cariri (UFCA). Endereço: Av. Tenente Raimundo Rocha $n^{\circ}$ 1639, Bloco I, Sala 405, Auditoria Interna, Bairro Cidade Universitária, Juazeiro do Norte/CE, Brasil, CEP: 63048-080. Telefone: (88) 3221-9490. E-mail: rafaelvalerio65@gmail.com.

https://orcid.org/0000-0001-9970-4953

${ }^{3}$ Doutora em Engenharia de Produção pela Universidade Federal de Santa Catarina (UFSC). Docente do Programa de Pós-Graduação em Administração e Controladoria da Universidade Federal do Ceará (UFC). Endereço: Av. da Universidade, 2431, Bairro Benfica, Fortaleza/CE, Brasil, CEP: 60020-180. Telefone: (85) 3366-7802. E-mail: alevasconcelos.ufc@gmail.com. https://orcid.org/0000-0002-6480-5620

Editor responsável pela aprovação do artigo: Dr. João Estevão Barbosa Neto Editora responsável pela edição do artigo: $\mathrm{Dr}^{\mathrm{a}}$. Bruna Camargos Avelino 
correlação significante entre a eficiência nos hospitais e a porcentagem de despesas custeadas por fontes de receitas próprias e do SUS. Além disso, observou-se uma diferença entre o nível de eficiência nos hospitais universitários de maior porte e o daqueles de menor porte.

Palavras-Chave: Eficiência, Atendimento assistencial, Hospitais universitários.

\title{
EFFICIENCY OF HEALTH AND SOCIAL CARE PROVISION AT UNIVERSITY HOSPITALS
}

\begin{abstract}
Developing countries have been strongly impacted by the worldwide rise in healthcare costs due to population aging, inflated medical costs, and economic and social polarization. Thus, public administrators, especially in the health sector, face the challenge of improving resource efficiency. At university hospitals, resource-efficient health and social care provision are further encumbered by research and teaching demands. Starting in 2011, the EBSERH (a Brazilian hospital management contractor) took over the management of a string of public hospitals, emphasizing efficiency. This study evaluated the efficiency of health and social care provision at 40 federal university hospitals managed by the EBSERH. Variables collected for 2018 were submitted to data envelopment analysis, outputoriented Banker-Charnes-Cooper modeling, and testing and correlation of means. Efficiency was confirmed for ten hospitals, six of which were located in Northeastern Brazil. The five least efficient hospitals included two facilities in Rio de Janeiro. Mortality was the variable with the most significant potential for improvement, suggesting managers give more attention to this issue. Hospital size was significantly associated with efficiency, but no significant correlation was found between efficiency and the percentage of expenses covered by the hospital's revenues vs. the Universal Health Care System (SUS).
\end{abstract}

Keywords: Efficiency, Health and social care, University hospitals.

\section{INTRODUÇÃO}

O valor despendido com saúde e o resultado desse dispêndio em termos de qualidade e eficiência constituem assuntos de grande relevância, tanto no segmento privado quanto na administração pública, dada a magnitude dos gastos no setor. Segundo relatório recente da Organização Mundial de Saúde (OMS), que contabiliza os gastos públicos e os privados, os custos mundiais com saúde já representam 10\% do Produto Interno Bruto (PIB) mundial. O aumento desses gastos é mais acelerado em países de baixo ou médio rendimento, onde os dispêndios com saúde crescem em média $6 \%$ a.a., enquanto nos países ricos o crescimento médio anual não passa de $4 \%$ (Xu et al., 2018).

No Brasil, o dispêndio total com saúde corresponde a 8,2\% do PIB, sendo $4,4 \%$ referentes a gastos privados e $3,8 \%$ alusivos a gastos públicos. A proporção de gastos públicos no país aproxima-se da média da América Latina e Caribe (3,6\% do PIB), mas ainda está longe de alcançar a média dos países da 
Organização para a Cooperação e Desenvolvimento Econômico (OCDE), de $6,5 \%$ do PIB (Tesouro Nacional, 2018). Mesmo nos países da OCDE, onde gastos com saúde representam um percentual maior do PIB, existe uma demanda crescente por mais investimentos no setor de saúde (Hadad, Hadad, \& SimonTuval, 2013).

Como visto, o desenvolvimento econômico demanda um gasto público maior em saúde, em razão do que, as questões referentes a sustentabilidade e melhoria de um sistema universal de saúde, como o Sistema único de Saúde (SUS), são destaques na agenda política dos países emergentes. Esse grupo de países enfrenta uma demanda crescente por serviços de saúde, agravada pelo aumento e envelhecimento populacional, somados a uma polarização econômica e social (Borgonovi \& Compagni, 2013).

Além da relevância dos gastos com saúde, Avelar, Silva e Fouto (2018) advertem que necessita-se levar em conta o impacto da Variação de Custos Médico-Hospitalares (VCMH) do Instituto de Estudos de Saúde Suplementar (IESS), que tem se apresentado continuamente maior do que a inflação da economia nacional. Peña (2008) acrescenta que há escassez de recursos tanto no segmento público quanto no privado, diante das exigências sempre crescentes. Esse cenário requer do gestor a busca da melhor combinação de recursos.

Segundo Farrell (1957), a eficiência em uma organização significa seu sucesso ao produzir o máximo possível mantendo-se as mesmas entradas, ou seja, utilizando os mesmos recursos. Logo, a permanente busca pela eficiência tornase uma condição para a sobrevivência da organização hospitalar.

Junto com a escassez de recursos, apontada por Peña (2008), outro fator complicador para a gestão das organizações do setor de saúde, e em especial para a gestão hospitalar, é a complexidade dessas instituições. As unidades hospitalares prestam serviços diversos, como diagnóstico, prevenção, tratamento, internação, educação e pesquisa (Silva, Costa, Abbas, \& Galdamez, 2017).

Corroborando a visão de Silva et al. (2017), Miranda, Carvalho, Martins e Faria (2007) destacam a heterogeneidade dos "produtos" ofertados pelas organizações hospitalares, sobretudo universitárias e de ensino, que além de ofertar serviços de saúde à população, são também responsáveis pela formação prática dos futuros profissionais de saúde. Lobo, Lins, Silva e Fiszman (2010) evidenciaram como sendo um desdobramento da complexidade dos hospitais universitários a existência de múltiplas dimensões dentro de cada uma dessas organizações. A citada obra identifica as seguintes dimensões: assistência, ensino e pesquisa.

Ao avaliar a integração das três citadas dimensões, Araújo e Leta (2014) identificaram que, na visão dos gestores dos hospitais universitários federais, ali prevalece o binômio ensino-assistência, em detrimento da pesquisa. Contudo, para a maioria dos gestores entrevistados pelos autores, a assistência (e não o ensino) foi evidenciada como a dimensão de maior peso.

No que tange à dimensão assistência, foco da presente investigação, os hospitais universitários federais são encarregados da oferta de serviços aos usuários em regime de internação, mediante ações que abrangem a promoção da saúde, a prevenção de agravos, o diagnóstico, o tratamento e a reabilitação 
(Lobo, Rodrigues, André, Azeredo, \& Lins, 2016). Desde a criação da EBSERH em 2011 (Lei n. 12.550, 201 1), os hospitais universitários federais vêm passando por uma reformulação no seu modelo de gestão. Ao analisar o processo de implementação da EBSERH, Borges, Barcelos e Rodrigues (2018) identificaram a empresa como um paradigma de gestão para o setor público. Nesse modelo de gestão empresarial, verifica-se uma acentuada pressão por eficiência, sendo o Estado visto como mais um agente da esfera econômica, historicamente considerado ineficiente e pouco produtivo.

Bonacim e Araújo (2010) observam que nos hospitais em geral o quadro de funcionários é financiado pelos seus instituidores, sendo, no caso dos hospitais universitários federais, o Ministério da Educação. Entretanto, o financiamento limitado às despesas de pessoal mostra-se insuficiente. Nesse contexto, o reembolso feito pelo SUS, com base em uma tabela que define um preço para cada procedimento, ou a obtenção de receitas próprias revestem-se de grande importância para garantir o seu regular funcionamento. Assim, dada a relevância da eficiência da gestão dos hospitais universitários federais, propõe-se o seguinte objetivo: avaliar a eficiência do atendimento assistencial nos hospitais universitários geridos pela EBSERH.

Como exposto, a eficiência é uma questão relevante para as organizações hospitalares. Entretanto, apesar de a DEA fornece informações sobre a eficiência técnica em hospitais, de maneira oposta ao que ocorre na literatura estrangeira, no Brasil não há disponibilidade de muitos estudos que façam uso dessa ferramenta (Silva, Moretti, \& Schuster, 2016).

No âmbito estrangeiro, Kohl, Schoenfelder, Fügener e Brunner (2019) procederam uma revisão das publicações que fizeram uso da DEA. O estudo em questão revisou 262 publicações que utilizaram a ferramenta para análise de dados no setor de saúde, com ênfase em hospitais. Os autores constataram que a relevância do assunto não se deve apenas à quantidade de estudos, já que também se identificou um número crescente de publicações.

Alguns estudos nacionais que fizeram uso da DEA para avaliar a eficiência nos hospitais universitários brasileiros (Araújo \& Leta, 2014; Lins, Lobo, Silva, Fiszman, \& Ribeiro, 2007; Lobo, Silva, Lins, \& Fiszman, 2009; Lobo et al., 2010, 2016; Lobo, Silva, Lins, Fiszman, \& Bloch, 2011 ; Peixoto, 2016). Vale destacar que a pesquisa de Lobo et al. (2009) representou uma importante contribuição para o estudo da eficiência nos hospitais universitários federais brasileiros ao utilizar a DEA para verificar a eficiência nas dimensões assistência e ensino, reconhecendo a importância de se verificar a eficiência de forma segregada, já que diferentes variáveis são consideradas no cálculo da eficiência de cada dimensão.

Com o advento da EBSERH em 2011, a dimensão assistência dos hospitais universitários ganhou relevância, já que após a mudança no modelo de gestão "os serviços assistenciais prestados por essas instituições à sociedade e sua capacidade de trabalho são criteriosamente redimensionados para atender às necessidades loco regionais e melhorar a sustentabilidade econômico-financeira desses hospitais" (Empresa Brasileira de Serviços Hospitalares [EBSERH], n.d.b).

Mais recentemente, Martini, Machado, Menezes e Souza (2019) avaliaram a eficiência com enfoque específico no aspecto financeiro. Na conclusão da pesquisa os autores recomendaram a realização de novos estudos utilizando a DEA para avaliar outras dimensões, como a assistência, inserindo-se novas 
variáveis no modelo. Sugere-se ainda como objeto de pesquisas futuras a avaliação da eficiência dos hospitais universitários sob o impacto da adesão à EBSERH (Martini et al., 2019; Peixoto, 2016).

Ante o exposto, observa-se uma lacuna que se pretende suprir com o presente estudo, já que se fará uso da DEA, ainda pouco utilizada no âmbito nacional para avaliação de hospitais universitários, com inserção de variáveis relevantes, a exemplo da taxa de ocupação hospitalar, procurando-se identificar a correlação entre os níveis de eficiência no atendimento assistencial e as respectivas fontes de financiamento de custeio.

\section{REFERENCIAL TEÓRICO}

Apresentam-se nesta seção os conceitos da eficiência na administração pública e no setor de saúde - sendo esta mensurada por meio da Análise Envoltória de Dados -, a EBSERH e a qualidade da assistência nos hospitais universitários federais e os estudos relacionados.

\subsection{Eficiência na Administração Pública e no Setor de Saúde}

O princípio da eficiência foi legalmente inserido na administração pública por meio da Emenda Constitucional n. 19, de 4 de junho de 1998 (Emenda Constitucional n. 19, 1998), por meio do qual buscou-se a implementação de um novo modelo de gestão que tivesse como foco aprimorar a aplicação de recursos escassos em busca de melhores resultados técnicos e operacionais, sem perder de vista a qualidade dos serviços ofertados.

Os primeiros estudos a discutir a eficiência na combinação dos fatores de produção foram desenvolvidos por Farrell (1957). No tocante à discussão sobre a eficiência na produção, esse autor foi pioneiro em destacar a importância de se medir a eficiência de uma indústria e de se identificar até que ponto uma determinada indústria pode aumentar a sua produção simplesmente aumentando sua eficiência, ou seja, sem consumir mais recursos.

Farrell (1957) inspirou o estudo seminal de Charnes, Cooper e Rhodes (1978), que introduziu a Análise por Envoltória de Dados (DEA, do inglês Data Envelopment Analysis), conhecida inicialmente como modelo CCR, em alusão aos seus idealizadores. Esse modelo inicial foi concebido para uma análise de retornos constantes de escala (Constant Returns to Scale - CRS). O modelo CCR é utilizado para análise de casos em que se tem uma proporcionalidade entre insumos (inputs) e produtos (outputs) (Peña, 2008; Sousa \& Ramos, 1999). Como uma evolução do modelo CCR, Banker, Charnes e Cooper (1984) propuseram um modelo de DEA que passou a considerar retornos variáveis de escala (Variable Returns to Scale - VRS), sendo conhecido como DEA-BCC.

No contexto da administração pública, a DEA foi apresentada por Peña (2008) como uma poderosa ferramenta para avaliar a eficiência técnica de unidades produtivas que usam insumos para geração de bens e serviços. Segundo o autor, a utilização da DEA é recomendada para pesquisas de eficiência na administração pública, já que tem sido aplicada de forma exitosa no estudo da eficiência de organizações públicas como escolas, universidades e estabelecimentos de saúde (hospitais e clínicas) (Peña, 2008). Destaca-se 
também estudos aplicados na alocação de recursos públicos no poder judiciário (Venturin, Souza, \& Bianchi, 2020) e na medição de eficiência dos clubes brasileiros de futebol (Dantas \& Boente, 2012; Nascimento, Nossa, Bermardes, \& Sousa, 2015).

A busca por eficiência no setor de saúde é pertinente, já que os prestadores precisam ser eficientes tanto no controle de custos quanto na qualidade dos serviços ofertados. No caso dos serviços públicos de saúde, faz-se necessário buscar sua maximização como premissa para utilização dos recursos públicos ou a minimização da utilização de recursos, mantidos os resultados predefinidos (Cesconetto, Lapa, \& Calvo, 2008).

Considerados relevantes componentes do setor de saúde, os hospitais constituem, segundo Kohl, Schoenfelder, Fügener e Brunner (2019), o principal fator de custos para os sistemas de saúde em todo o mundo, e enfrentam uma pressão crescente para melhorar sua eficiência. Entretanto, estimar a eficiência hospitalar não é tarefa simples. Dentre várias ferramentas, a DEA ganha destaque na avaliação da eficiência das organizações prestadoras de serviços de saúde (Kohl et al., 2019).

Vale destacar o estudo realizado por Espejo, Portulhak e Martins (2015), que investigaram as práticas de controle gerencial utilizadas pelos hospitais universitários federais, averiguando o porte como um fator explicativo do grau de adoção de tais práticas. Concluíram que o desenvolvimento do controle gerencial foi mais avançado em hospitais de grande porte.

Devido à relevância de sua atuação, os hospitais universitários federais têm sido objeto de estudos voltados para compreender aspectos de sua gestão. Segundo Borges et al. (2018), a criação da EBSERH em 2011 projetou a adoção de um modelo de gestão empresarial nos hospitais universitários federais.

\subsection{A EBSERH e a Qualidade da Assistência nos Hospitais Universitários Federais}

De acordo com o Conselho Federal de Medicina, "os hospitais universitários são centros de formação de recursos humanos e de desenvolvimento de tecnologia para a área de saúde" (Conselho Federal de Medicina [CFM], 2019). O CFM destaca também que por meio da oferta de serviços à população, os hospitais universitários fazem o aprimoramento permanente do atendimento e elaboram protocolos técnicos para diversas patologias.

Os hospitais universitários federais exercem um relevante papel no setor de saúde, ao atuar concomitantemente como importantes centros de formação de recursos humanos na área de saúde, apoiando as atividades de ensino, pesquisa e extensão desenvolvidas pelas universidades federais brasileiras (EBSERH, n.d.c). Além da dimensão ensino, os hospitais universitários também exercem papel de relevância na dimensão assistência.

A partir de 2004, a implementação da Política de Restruturação dos Hospitais de Ensino deu início a uma integração mais orgânica das dimensões ensino e assistência. Nesse período iniciou-se um processo de certificação dos hospitais universitários e de ensino, por meio de visitas bianuais de representantes do MEC e do Ministério da Saúde (MS). Por meio dessas visitas, passou-se a se 
verificar quesitos relacionados à integração docente-assistencial, integração ao SUS e qualidade de gestão (Lobo et al., 2010).

De acordo com a EBSERH (n.d.b), a rede de hospitais universitários federais reúne 50 hospitais, vinculados a 35 universidades federais, presentes nas cinco regiões brasileiras. Na esfera da assistência à saúde, esses hospitais são centros de referência de média e alta complexidade para O SUS. Dos 50 hospitais universitários, 40 possuem contrato com a EBSERH, o que demonstra a abrangência da atuação do novo modelo de gestão inaugurado com a criação da empresa.

A criação da EBSERH foi autorizada pela Lei n. 12.550, de 15 de dezembro de 2011 (Lei n. 12.550, 201 1), tendo como finalidade:

a prestação de serviços gratuitos de assistência médico-hospitalar, ambulatorial e de apoio diagnóstico e terapêutico à comunidade, assim como a prestação às instituições públicas federais de ensino ou instituições congêneres de serviços de apoio ao ensino, à pesquisa e à extensão, ao ensino-aprendizagem e à formação de pessoas no campo da saúde pública, observada, nos termos do art. 207 da Constituição Federal, a autonomia universitária.

Diante do contexto de implantação da EBSERH, um importante aspecto da dimensão assistência é o aparente confronto entre dois modelos de gestão. A EBSERH trouxe um modelo de gestão mais alinhado a uma lógica gerencial própria de empresa privada. Esse alinhamento gerencial com a administração privada pode gerar um confronto com princípios propostos pela gestão do SUS, dentre eles a integralidade da assistência (Borges et al., 2018).

Espejo et al. (2015) reiteram a relevância da atuação dos hospitais universitários federais por meio do SUS, e ressaltam a importância do desenvolvimento de pesquisas sobre práticas de controle gerencial nos estabelecimentos, devido ao seu relevante papel para o SUS, sobretudo na realização de procedimentos de média e alta complexidade. Também merece destaque a atuação dessas organizações na formação de profissionais na área de saúde, conforme estipula a lei que autorizou a criação da EBSERH.

\subsection{Estudos Abordando Eficiência nos Hospitais Universitários}

No âmbito nacional foram identificados alguns estudos que fizeram uso da DEA para verificar a eficiência nos hospitais universitários federais brasileiros. Lins et al. (2007) foram pioneiros ao demonstrar a viabilidade de utilização da DEA, ao verificar a eficiência de 31 hospitais universitários federais. Para tanto, utilizaram variáveis relacionadas à dimensão assistência e variáveis ligadas às dimensões ensino e pesquisa, como, por exemplo, o número de estudantes de pósgraduação e o número de programas de pós-graduação em medicina. Os autores calcularam o nível de eficiência para cada uma das três dimensões, concluindo que na dimensão assistência 17 hospitais revelaram-se eficientes.

Com o objetivo de avaliar o impacto da reforma de financiamento na produtividade, Lobo et al. (2009), mediante aplicação da DEA, construíram fronteiras de eficiência. Para identificar as mudanças de desempenho 
decorrentes da reforma de financiamento, os autores calcularam o Índice de Malmquist para identificar mudanças de desempenho ao longo do quadriênio 2003-2006. Concluiu-se que a reforma de financiamento possibilitou o desenvolvimento de melhorias gerenciais, ponderando-se, porém, que seria necessária uma observação por um período maior. De maneira complementar, os resultados indicam que uma das principais razões para a reforma do modelo de financiamento foi a necessidade de reduzir as ineficiências de atenção de saúde prestada pelos hospitais de ensino (Lobo et al., 2009).

Seguindo abordagem semelhante, Lobo et al. (2010) avaliaram o desempenho e a integração das dimensões assistência e ensino nos hospitais universitários federais, por meio da aplicação da DEA em rede (network DEA), sinalizando para a importância da inclusão da dimensão qualidade na avaliação da eficiência dos hospitais. Lobo et al. (2016) também utilizaram a variação da DEA em rede. Essa variação considera variáveis de ligação que pertencem a mais de uma dimensão. Tais variáveis são utilizadas para analisar o deslocamento da fronteira de eficiência entre dois períodos, no caso do estudo, 2010 e 2013.

Foram ainda identificados alguns estudos sobre a eficiência nos hospitais universitários considerando mais de uma dimensão (assistência, ensino e pesquisa) ou somente em relação ao aspecto financeiro (Martini et al., 2019). Martini et al. (2019) avaliaram os hospitais universitários utilizando uma amostra censitária, abrangendo o quinquênio 2013-2017. Esse estudo contemplou somente $\mathrm{o}$ aspecto financeiro, utilizando-se variáveis como recursos financeiros totais e valores do faturamento dos serviços ofertados no âmbito do SUS. Os resultados indicaram que os hospitais universitários da UFJF e da UFMA, o HUWC, da UFC, e o HESFA, da UFRJ foram considerados eficientes em todo o período considerado.

Peixoto (2016) também fez uso da DEA para avaliar os hospitais universitários federais. Com base nos dados de 2014, a autora definiu as variáveis com foco na produção de residentes nas diversas especialidades médicas, sendo essa a variável utilizada como output no estudo.

O presente estudo diferencia-se dos anteriores, portanto, por direcionar o foco para a dimensão assistência e por utilizar indicadores de gestão e informações orçamentárias concernentes ao atendimento assistencial dos hospitais universitários federais, que retratam o desempenho também sob outros aspectos relevantes, como a qualidade, alinhado às recomendações de Lobo et al. (2010). Ademais, este estudo inova ao analisar o relacionamento entre a eficiência do atendimento assistencial e as fontes de financiamento do custeio dos hospitais universitários geridos pela EBSERH, por meio da proporção das receitas próprias e do SUS, em relação ao valor das despesas empenhadas (EBSERH, n.d.a), variável essa que até o momento não foi aplicada em outros estudos.

\section{PROCEDIMENTOS METODOLÓGICOS}

No que tange ao enquadramento metodológico, o presente estudo possui natureza quantitativa. Quanto aos objetivos, classifica-se como descritivo, no qual se fez uso da análise documental ao se proceder à coleta de dados nos websites da EBSERH e do Departamento de Informática do SUS (DATASUS). 
Utilizaram-se como base de dados indicadores de gestão e informações orçamentárias, divulgados por meio dos painéis de monitoramento disponibilizados pela EBSERH. Esses painéis fazem parte de uma iniciativa de transparência adotada pela instituição, que faz uso de uma solução de B.l. (Business Intelligence), para divulgar uma gama de informações acerca da execução orçamentária e indicadores financeiros, operacionais, de qualidade e outros (EBSERH, n.d.a). Também foram extraídos dados do Cadastro Nacional de Estabelecimentos de Saúde (CNES) e do tabulador de dados TABNET, mantidos pelo DATASUS (Ministério da Saúde, n.d.). A Figura 2 detalha as variáveis (inputse outputs) utilizadas na DEA do estudo, indicando a fonte de dados e as referências de cada uma delas.

A coleta ocorreu em dezembro de 2019, sendo utilizados os dados referentes ao exercício de 2018, por ser, no período da coleta, o mais recente com dados completos. No período da coleta o painel de indicadores da EBSERH apresentou dados incompletos para os indicadores referentes ao exercício de 2017, ano inicial de divulgação dos dados.

A amostra utilizada na pesquisa é censitária, ou seja, considerou todos os 40 hospitais universitários federais que possuíam contrato com a EBSERH em 2018. A Figura 1 apresenta as DMUs (Decision Making Units) utilizadas no estudo, distribuídas por estado.

\begin{tabular}{|c|c|c|}
\hline $\begin{array}{l}\mathrm{N}^{\circ} \text { de } \\
\text { Ordem }\end{array}$ & Hospital Universitário & Localização \\
\hline 1 & Hospital Universitário de Brasília (HUB-UnB) & Distrito Federal \\
\hline 2 & Hospital das Clínicas da UFG (HC-UFG) & Goiás \\
\hline 3 & Hospital Universitário Júlio Müller (HUJM-UFMT) & Mato Grosso \\
\hline 4 & Hospital Universitário da UFGD (HU-UFGD) & Mato Grosso do Sul \\
\hline 5 & $\begin{array}{l}\text { Hospital Universitário Maria Aparecida Pedrossian (HUMAP- } \\
\text { UFMS) }\end{array}$ & Mato Grosso do Sul \\
\hline 6 & Hospital Universitário Professor Alberto Antunes (HUPAA-UFAL) & Alagoas \\
\hline 7 & Hospital Universitário Professor Edgard Santos (HUPES-UFBA) & Bahia \\
\hline 8 & Maternidade Climério de Oliveira (MCO-UFBA) & Bahia \\
\hline 9 & Hospital Universitário Wálter Cantídio (HUWC-UFC) & Ceará \\
\hline 10 & Maternidade Escola Assis Chateaubriand (MEAC-UFC) & Ceará \\
\hline 11 & Hospital Universitário da UFMA (HU-UFMA) & Maranhão \\
\hline 12 & Hospital Universitário Lauro Wanderley (HULW-UFPB) & Paraíba \\
\hline 13 & Hospital Universitário Alcides Carneiro (HUAC-UFCG) & Paraíba \\
\hline 14 & Hospital Universitário Júlio Bandeira (HUJB-UFCG) & Paraíba \\
\hline 15 & Hospital das Clínicas da UFPE (HC-UFPE) & Pernambuco \\
\hline 16 & Hospital Universitário da UNIVASF (HU-UNIVASF) & Pernambuco \\
\hline 17 & Hospital Universitário da UFPI (HU-UFPI) & Piauí \\
\hline 18 & Hospital Universitário Ana Bezerra (HUAB-UFRN) & $\begin{array}{l}\text { Rio Grande do } \\
\text { Norte }\end{array}$ \\
\hline 19 & Hospital Universitário Onofre Lopes (HUOL-UFRN) & $\begin{array}{l}\text { Rio Grande do } \\
\text { Norte }\end{array}$ \\
\hline 20 & Maternidade Escola Januário Cicco (MEJC-UFRN) & $\begin{array}{l}\text { Rio Grande do } \\
\text { Norte }\end{array}$ \\
\hline 21 & Hospital Universitário de Lagarto (HUL-UFS) & Sergipe \\
\hline 22 & Hospital Universitário de Sergipe (HUS-UFS) & Sergipe \\
\hline 23 & Hospital Universitário Getúlio Vargas (HUGV-UFAM) & Amazonas \\
\hline 24 & Hospital Universitário João de Barros Barreto (HUJBB-UFPA) & Pará \\
\hline 25 & Hospital Universitário Bettina Ferro de Souza (HUBFS-UFPA) & Pará \\
\hline
\end{tabular}




\begin{tabular}{|l|l|l|}
\hline $\begin{array}{c}\text { No de } \\
\text { Ordem }\end{array}$ & \multicolumn{1}{|c|}{ Hospital Universitário } & \multicolumn{1}{|c|}{ Localização } \\
\hline 26 & Hospital de Doenças Tropicais (HDT-UFT) & Tocantins \\
\hline 27 & Hospital Universitário Cassiano Antônio Moraes (HUCAM-UFES) & Espírito Santo \\
\hline 28 & Hospital das Clínicas da UFMG (HC-UFMG) & Minas Gerais \\
\hline 29 & Hospital de Clínicas de Uberlândia (HCU-UFU) & Minas Gerais \\
\hline 30 & Hospital de Clínicas da UFTM (HCU-UFTM) & Minas Gerais \\
\hline 31 & Hospital Universitário de Juiz de Fora (HUJF-UFJF) & Minas Gerais \\
\hline 32 & Hospital Universitário Gaffrée e Guinle (HUGG-UNIRIO) & Rio de Janeiro \\
\hline 33 & Hospital Universitário Antônio Pedro (HUAP-UFF) & Rio de Janeiro \\
\hline 34 & $\begin{array}{l}\text { Hospital Universitário Professor Horácio Carlos Panepucci } \\
\text { (HUPHCP-UFSCAR) }\end{array}$ & São Paulo \\
\hline 35 & Complexo Hospital de Clínicas (CHC-UFPR) & Paraná \\
\hline 36 & Maternidade Víctor Ferreira do Amaral (MVFA-UFPR) & Paraná \\
\hline 37 & Hospital Escola da UFPEL (HE-UFPEL) & Rio Grande do SUl \\
\hline 38 & Hospital Universitário de Santa Maria (HUSM-UFSM) & Rio Grande do SUl \\
\hline 39 & $\begin{array}{l}\text { Hospital Universitário Doutor Miguel Riet Correa Júnior } \\
\text { (HUDMRCJ-FURG) }\end{array}$ & Rio Grande do Sul \\
\hline 40 & $\begin{array}{l}\text { Hospital Universitário Professor Polydoro Ernâni de São } \\
\text { Thiago (HUPPEST-UFSC) }\end{array}$ & Santa Catarina \\
\hline
\end{tabular}

Figura 1 - Relação de DMUs

Fonte: EBSERH (n.d.a).

A DEA tem sido utilizada para analisar o desempenho de DMUs que utilizam os mesmos tipos de insumo (inputs) para produzir os mesmos bens e/ou serviços (outputs). Os hospitais universitários, portanto, podem ser enquadrados no conceito de DMU, como demonstrado por Lins et al. (2007).

De acordo com Mello et al. (2005), para aplicação da DEA, faz-se necessária a implementação de três etapas. A primeira delas consiste em selecionar e definir as DMUs, no caso do estudo os hospitais universitários geridos pela EBSERH. A segunda etapa compreende a seleção das variáveis utilizadas na DEA. A seleção das variáveis adotadas na pesquisa considerou sua adoção em estudos anteriores da área (Figura 2), sendo os dados referentes a 2018. A terceira etapa é a seleção e aplicação do modelo.

A técnica tem como pressupostos o modelo CCR, concebido para analisar os retornos constantes de escala (Constant Returns to Scale-CRS), idealizado por Charnes, Cooper e Rhodes (1978) e o BCC, proposto por Banker, Charnes e Cooper (1984), passando a considerar retornos variáveis de escala (Variable Returns to Scale - VRS) (Peña, 2008; Sousa \& Ramos, 1999). O resultado obtido a partir do modelo varia entre 0 e 1, em que " 0 " indica que a DMU é ineficiente, e "I" significa que a DMU é eficiente (Afonso, Schuknecht, \& Tanzi, 2010). Nesta pesquisa optou-se por utilizar o modelo DEA-BCC, com orientação para outputs, de Banker et al. (1984), que considera retornos variáveis de escala.

As variáveis elencadas na Figura 2 foram utilizadas para o cálculo da eficiência do atendimento assistencial de cada hospital universitário, com suporte do software Frontier Analyst 4. No processamento da DEA, foi adotado o modelo DEA-BCC, com orientação para outputs, já que se pretende, a partir dos mesmos níveis de insumos, a obtenção dos melhores resultados. 


\begin{tabular}{|c|c|c|c|c|}
\hline Variável & Descrição & Tipo & Fonte & Referência \\
\hline NLeitos & Número total de leitos & \multirow[t]{2}{*}{ Input } & CNES-DATASUS & $\begin{array}{l}\text { Cesconetto et al. (2008) } \\
\text { Kirigia, Emrouznejad, } \\
\text { Sambo, Munguti e Liambila } \\
\text { (2004) } \\
\text { Tonelotto, Crozatti, Moraes } \\
\text { e Righetto (2019) }\end{array}$ \\
\hline TGastos & $\begin{array}{lll}\text { Valor } & \text { total } & \text { das } \\
\text { despesas } & \text { empenhadas }\end{array}$ & & EBSERH & $\begin{array}{l}\text { Silva et al. (2017) } \\
\text { Tonelotto et al. (2019) }\end{array}$ \\
\hline Inter & Total de internações & \multirow{4}{*}{ Output } & TABNET-DATASUS & $\begin{array}{l}\text { Lins et al. (2007) } \\
\text { Lobo et al. (2010) } \\
\text { Lobo et al. (2011) }\end{array}$ \\
\hline TxOcup & $\begin{array}{l}\text { Taxa de ocupação } \\
\text { média }\end{array}$ & & EBSERH & Cesconetto et al. (2008) \\
\hline IPerm & $\begin{array}{l}\text { Inverso da taxa de } \\
\text { permanência }\end{array}$ & & TABNET-DATASUS & $\begin{array}{llll}\text { Souza, Nishijima e } & \text { Rocha } \\
(2010) & & \\
\end{array}$ \\
\hline Imort & $\begin{array}{l}\text { Inverso da taxa de } \\
\text { mortalidade média }\end{array}$ & & TABNET-DATASUS & $\begin{array}{l}\text { Tonelotto et al. (2019) } \\
\text { Souza et al. (2010) }\end{array}$ \\
\hline
\end{tabular}

Figura 2 - Variáveis utilizadas no estudo

Fonte: Elaborada pelos autores.

Com o intuito de se agrupar as DMUs por nível de eficiência, na sequência foi realizada uma análise de c/usters, ou análise de agrupamentos, que constitui um método empírico e objetivo para realizar uma das tarefas mais naturais dos seres humanos - classificação (Hair, Black, Babin, Anderson, \& Tatham, 2009). Assim, por meio dessa técnica procurou-se promover o agrupamento dos hospitais universitários adotando como base o nível de eficiência calculado por meio da DEA, sendo possível a formação de quatro grupos: eficiência baixa, eficiência média baixa, eficiência média alta e eficiência alta.

A partir desse ponto, realizou-se o teste de correlação de Spearman, que considerou o nível de eficiência e a porcentagem de participação de receitas próprias e do SUS no custeio dos hospitais universitários, com dados de 2018. Essa porcentagem é disponibilizada pela EBSERH, em seu painel de indicadores da EBSERH, e se refere à proporção das receitas próprias e do SUS em relação ao valor das despesas empenhadas, exceto os recursos de custeio relacionados ao pagamento da folha de pessoal, sentenças e bolsas de residências multiprofissionais (EBSERH, n.d.a). Cabe informar que a exclusão das despesas de pessoal, sentenças e bolsas para fins do valor do indicador TGastos é feita pela própria EBSERH, conforme Painel de Informações Orçamentárias e Financeiras da organização.

No mesmo sentido, adotou-se o teste de diferenças entre médias não paramétrico de Mann-Whitney. Esse teste é aplicado para testar se duas amostras são provenientes da mesma população. De acordo com o Ministério da Saúde (1977) os hospitais podem ser classificados, segundo o seu porte em pequeno, médio, grande e especial. Em razão da existência de quatro diferentes portes dos hospitais, o teste de diferença de médias foi realizado em pares. Cabe mencionar que antes da aplicação do teste não paramétrico foi realizado o teste de normalidade, segundo o qual os dados não apresentam distribuição normal (pvalor inferior ao nível de significância de $5 \%$ ). Ademais, para a análise dos dados por meio do método DEA, foi utilizado o software Frontier Analyst, versão 4, 
enquanto para a realização das análises estatísticas utilizou-se o IBM SPSS Statistics 20.

\section{ANÁLISE E DISCUSSÃO DOS RESULTADOS}

Preliminarmente foi realizada uma análise descritiva das variáveis. Esse tipo de análise possibilita uma melhor compreensão do comportamento dos dados, identificando-se tendências, variabilidade e valores atípicos (Fávero, Belfiore, Silva, \& Chan, 2009). A Tabela 1 apresenta a estatística descritiva de cada variável utilizada na DEA.

Tabela 1

Estatística descritiva das variáveis do estudo

\begin{tabular}{|c|c|c|c|c|c|}
\hline Variável & Mínimo & Máximo & Média & Desvio-padrão & $\begin{array}{l}\text { Coeficiente de } \\
\text { variação (\%) }\end{array}$ \\
\hline Nleitos & 31 & 653 & 222 & 142 & 63,84 \\
\hline TGastos (R\$) & $5.525 .008,00$ & $489.363 .741,00$ & $156.756 .834,00$ & $127.041 .330,00$ & 81,04 \\
\hline Inter & 279 & 22.193 & 8.271 & 5.437 & 65,74 \\
\hline TxOcup & 19 & 140 & 70,15 & 21,52 & 30,67 \\
\hline Perm & 1 & 18 & 6,97 & 2,51 & 35,98 \\
\hline lort & 0 & 14 & 3,69 & 2,89 & 78,26 \\
\hline
\end{tabular}

Legenda: NLeitos: Número total de leitos, TGastos: Valor total das despesas empenhadas; Inter: Total de internações; TxOcup: Taxa de ocupação média; Perm: Taxa de permanência; Mort: Taxa de mortalidade média.

Fonte: Elaborada pelos autores.

O Ministério da Saúde (1977) classificou os hospitais de acordo com os respectivos portes, adotando como base o número de leitos. Considerando-se essa classificação, a rede de hospitais universitários que possuem contrato com a EBSERH compõe-se de unidades de portes heterogêneos, como pode ser constatado na Tabela 1. Segundo os parâmetros do Ministério da Saúde (1977), entre as 40 DMUs da amostra, há hospitais de pequeno porte (até 50 leitos), médio porte (51 a 150 leitos), grande porte (151 a 500 leitos) e de porte especial (acima de 500 leitos). O número de leitos varia de 31, no caso do HUBFS-UFPA, a 653, caso do CHC-UFPR.

O total de gastos corresponde ao valor das despesas empenhadas pelos hospitais universitários durante o exercício de 2018, variando de $\mathrm{R} \$ 5.525 .008,00$ (HUJB-UFCG) a R\$ 489.363.741,00 (HCU-UFU). Os valores máximo e mínimo dos gastos dos hospitais universitários em 2018 reforçam a heterogeneidade dos dados utilizados na DEA, o que também é evidenciado pelo confronto entre os valores extremos das outras variáveis. Na Tabela 1, observa-se, portanto, que as variáveis utilizadas na DEA apresentam dados heterogêneos, sendo o total de gastos, que representa o valor das despesas empenhadas, a variável com maior coeficiente de variação.

Outra variável que apresenta um alto coeficiente de variação é a taxa de mortalidade. Chama a atenção o fato de que os dois hospitais que apresentam a maior e a menor taxa de mortalidade são ambos vinculados à UFPA. Enquanto o HUBFS apresentou a menor taxa de mortalidade da amostra em 2018 (nula, no caso), O CHUJBB assinalou a maior (14\%). Vale destacar que a taxa de 
mortalidade é obtida pelo quociente entre o número de óbitos de pacientes com mais de 24h de internação e o número de saídas no período, multiplicando-se o resultado por 100. A variável monitora a qualidade do atendimento assistencial objetivando o planejamento de ações que contribuam para uma maior efetividade e eficiência do cuidado com a saúde (EBSERH, n.d.a) mas apresenta limitação em função da complexidade.

Com o objetivo de identificar eventuais redundâncias entre as variáveis, foi realizado teste de correlação entre as variáveis utilizadas no modelo DEA, apresentadas na Figura 2. A Tabela 2 apresenta o resultado do teste de correlação de Pearson.

\section{Tabela 2}

Correlação entre as variáveis do estudo

\begin{tabular}{|c|c|c|c|c|c|c|}
\hline \multirow{2}{*}{ Variável } & \multicolumn{2}{|c|}{ Input } & \multicolumn{4}{|c|}{ Output } \\
\hline & Nleitos (I) & TGastos (I) & Inter (O) & TxOcup (O) & (Perm (O) & IMort (O) \\
\hline Nleitos (I) & 1 & & & & & \\
\hline TGastos (I) & $0,864^{* *}$ & 1 & & & & \\
\hline Inter (O) & $0,819^{* *}$ & $0,739 * *$ & 1 & & & \\
\hline TxOcup (O) & 0,224 & 0,112 & $0,483^{* *}$ & 1 & & \\
\hline IPerm (O) & $-0,355^{*}$ & $-0,192$ & $-0,006$ & $-0,235$ & 1 & \\
\hline IMort (O) & $-0,330^{*}$ & $-0,214$ & $-0,070$ & $-0,259$ & $0,641^{* *}$ & 1 \\
\hline
\end{tabular}

Legenda: NLeitos: Número total de leitos, TGastos: Valor total das despesas empenhadas; Inter: Total de internações; TxOcup: Taxa de ocupação média; Perm: Taxa de permanência; Mort: Taxa de mortalidade média.

Nota: (**) Significância estatística dos fatores ao nível de 1\%; (*) Significância estatística dos fatores ao nível de $5 \%$.

Fonte: Elaborada pelos autores.

De acordo com a Tabela 2, as variáveis TGastos e NLeitos, ambas utilizadas como inputs, apresentaram correlação considerada alta. Peixoto (2016) atenta que é desejável que se tenha uma correlação reduzida entre os fatores de input. No entanto, assim como Peixoto (2016), não se assumiu o atendimento a esse quesito como fundamental para o presente estudo, dada a relevância que o número de leitos e os recursos financeiros possuem para a atividade hospitalar. As duas variáveis em questão são amplamente utilizadas em estudos que avaliam a eficiência hospitalar (Kohl et al., 2019). Segundo Lee e Kim (2018), o número de leitos é um importante indicador porque representa o nível de infraestrutura de saúde. A falta de leitos pode afetar o número de internações, ocasionando a superlotação.

Conforme Tabela 2, as variáveis NLeitos e Inter apresentaram correlação alta, o que era esperado. Os hospitais que dispõem de uma quantidade maior de leitos, a priori, permitem um número maior de internações. A seguir, a Tabela 3 apresenta o resultado do cálculo da eficiência de cada DMU, realizado por meio da DEA, e respectiva classificação no ranking de eficiência. 
Tabela 3

Eficiência dos hospitais da amostra

\begin{tabular}{cccccc}
\hline Ranking & DMU & Nível de eficiência & Ranking & DMU & Nível de eficiência \\
\hline $1^{\circ}$ & HU-UNIVASF & 100,00 & $21^{\circ}$ & HC-UFPE & 84,52 \\
$1^{\circ}$ & CHC-UFPR & 100,00 & $22^{\circ}$ & HDT-UFT & 82,46 \\
$1^{\circ}$ & HCU-UFU & 100,00 & $23^{\circ}$ & HUB-UnB & 75,93 \\
$1^{\circ}$ & HUAB-UFRN & 100,00 & $24^{\circ}$ & HUPHCP-UFSCAR & 74,87 \\
$1^{\circ}$ & HUBFS-UFPA & 100,00 & $25^{\circ}$ & HUWC-UFC & 73,50 \\
$1^{\circ}$ & HUJB-UFCG & 100,00 & $26^{\circ}$ & HULW-UFPB & 69,38 \\
$1^{\circ}$ & HU-UFMA & 100,00 & $27^{\circ}$ & HUPES-UFBA & 66,49 \\
$1^{\circ}$ & MEAC-UFC & 100,00 & $28^{\circ}$ & MCO-UFBA & 64,11 \\
$1^{\circ}$ & MEJC-UFRN & 100,00 & $29^{\circ}$ & HE-UFPEL & 62,64 \\
$1^{\circ}$ & MVFA-UFPR & 100,00 & $30^{\circ}$ & HU-UFPI & 61,87 \\
$11^{\circ}$ & HCU-UFTM & 99,25 & $31^{\circ}$ & HUJF-UFJF & 59,58 \\
$12^{\circ}$ & HUMAP-UFMS & 98,19 & $32^{\circ}$ & HUPAA-UFAL & 59,55 \\
$13^{\circ}$ & HC-UFMG & 96,31 & $33^{\circ}$ & HUJM-UFMT & 59,30 \\
$14^{\circ}$ & HU-UFGD & 91,96 & $34^{\circ}$ & HUDMRCJ-FURG & 57,64 \\
$15^{\circ}$ & HUSM-UFSM & 90,61 & $35^{\circ}$ & HUAC-UFCG & 57,31 \\
$16^{\circ}$ & HC-UFG & 90,30 & $36^{\circ}$ & HUGG-UNIRIO & 56,32 \\
$17^{\circ}$ & HUCAM-UFES & 88,73 & $37^{\circ}$ & HUAP-UFF & 55,00 \\
$18^{\circ}$ & HUPPEST-UFSC & 86,24 & $38^{\circ}$ & HUGV-UFAM & 51,44 \\
$19^{\circ}$ & HUL-UFS & 86,16 & $39^{\circ}$ & HUS-UFS & 50,94 \\
$20^{\circ}$ & HUOL-UFRN & 85,39 & $40^{\circ}$ & HUJBB-UFPA & 36,85 \\
\hline
\end{tabular}

Fonte: Elaborada pelos autores.

De acordo com a Tabela 3, o cálculo apontou que 10 hospitais universitários alcançaram a fronteira de eficiência. Dentre as DMUs eficientes, estão presentes seis hospitais da Região Nordeste; dois da Região Sul, ambos vinculados à UFPR; um da Região Sudeste e um da Região Norte, no caso o HUBFSUFPA, cuja posição provavelmente está relacionado ao fato de apresentar a menor taxa de mortalidade da amostra, como já antes observado.

Usando DMUs distintas, Martini et al. (2019) avaliaram a eficiência dos hospitais universitários no aspecto financeiro no exercício de 2017. Identificaram que 21 dos 48 hospitais analisados atingiram a fronteira de eficiência empregando variáveis como recursos financeiros totais e valores do faturamento dos serviços ofertados no âmbito do SUS. Apesar das diferenças nas DMUs estudadas e nas variáveis adotadas nesta pesquisa, dos 10 hospitais que atingiram a eficiência de $100 \%$ no presente estudo, sete também foram apontados como eficientes por Martini et al. (2019): CHC-UFPR, HCU-UFU, HUUFMA, HUAB-UFRN, HUBFS-UFPA, MEJC-UFRN e MVFA-UFPR.

Pode-se conjecturar que os hospitais universitários classificados como eficientes na dimensão assistência e no aspecto financeiro podem ser considerados como referências de bom desempenho, tomando-se por base os resultados das duas pesquisas. Há que se fazer uma ressalva em relação ao fato de as duas pesquisas terem utilizado dados de exercícios diferentes (2017 e 2018) e amostras ligeiramente divergentes no que se refere às DMUs analisadas.

Dois hospitais que atingiram a fronteira de eficiência também foram apontados como eficientes por Lobo et al. (2016), que utilizaram dados referentes aos exercícios do quadriênio 2010-2013, e calcularam a eficiência para as dimensões assistência, ensino e pesquisa. O HCU-UFU e o HU-UFMA atingiram a 
fronteira de eficiência no mencionado estudo tanto na dimensão assistência como no escore total, que considerou as três citadas dimensões.

O HCU-UFU, além de ter sido classificado como eficiente neste estudo e por Lobo et al. (2016) e Martini et al. (2019), também atingiu a fronteira de eficiência em outros estudos, como os de Lins et al. (2007) e Lobo et al. (2009), no que tange à dimensão assistência. Por ter sido identificado como eficiente em estudos que utilizaram dados de diferentes períodos, bem como variáveis distintas, o HCU-UFU se destaca dos demais, podendo ser apontado como referência para outros hospitais universitários. Nesse ponto, cabe ressaltar que o HCU-UFU registra o maior volume de despesas empenhadas.

Dentre as DMUs eficientes, constam hospitais de grande porte, a exemplo do HCU-UFU, que possui 506 leitos, e do CHC-UFPR, que, com 653 leitos, é o maior da amostra; e hospitais de pequeno porte, como o HUBFS-UFPA, que registrou 0 menor volume de gastos em 2018.

Dentre os cinco hospitais universitários com os menores níveis de eficiência, dois deles ficam no Rio de Janeiro (HUAP-UFF e HUGG-UNIRIO), valendo notar que o estudo abrangeu apenas os hospitais geridos pela EBSERH. Embora os demais hospitais universitários do Rio de Janeiro não tenham sido avaliados, em função do critério adotado na seleção da amostra, o baixo nível de eficiência das duas citadas unidades desperta para a necessidade de maior atenção em relação à qualidade do seu atendimento assistencial.

O HUL-UFS também apresentou um baixo nível de eficiência. Dentre as unidades que apresentaram os mais baixos níveis de eficiência, esse possui o menor porte, com apenas 78 leitos e gasto total de $\mathrm{R} \$ 18.358 .608,77$ em 2018. Uma unidade apontada como referência para essa DMU, por ter porte semelhante, foi o HUAB-UFRN (63 leitos e gastos de R\$11.797.036,74), que, apesar disso, atingiu a fronteira de eficiência. A diferença entre os desempenhos das duas unidades ocorre, principalmente, por causa da taxa de mortalidade. Enquanto em 20180 HUL-UFS assinalou 12,54, o HUAB-UFRN assinalou 0,24.

O HUJBB-UFPA foi a DMU com pior desempenho. Vale destacar que os valores máximos de tempo médio de permanência hospitalar (IPerm) e taxa de mortalidade (Mort), mostrados na Tabela 1, são desse hospital universitário, o que explica o fato de ele ter apresentado o mais baixo nível de eficiência dentre todas as unidades avaliadas.

Em Martini et al. (2019), sobre a eficiência com enfoque no aspecto financeiro considerando diferentes DMUs e adotando outras variáveis, na análise do período 2013-2017 e do exercício de 2017, isoladamente, O HUJBB-UFPA também figura em uma das últimas posições no que tange ao nível de eficiência.

Além de calcular a eficiência das DMUs, a DEA possibilita identificar os seus potenciais de melhoria, ou seja, as alterações que precisam ser implementadas para que as unidades classificadas como ineficientes possam atingir a fronteira de eficiência. A Tabela 4 mostra, em porcentagens, os potenciais de melhoria de toda a amostra. 
Tabela 4

Potenciais de melhoria das DMUs

\begin{tabular}{lcc}
\hline & Variável & Potencial de melhoria (\%) \\
\hline Nleitos (I) & $-0,33$ \\
TGastos (I) & $-0,92$ \\
Inter (O) & 5,94 \\
TxOcup (O) & 2,13 \\
IPerm (O) & 2,95 \\
IMort (O) & 87,73
\end{tabular}

Legenda: NLeitos: Número total de leitos; TGastos: Valor total das despesas empenhadas; Inter: Total de internações; TxOcup: Taxa de ocupação média; IPerm: Inverso da taxa de permanência; IMort: Inverso da taxa de mortalidade média; (I): Inputs ou entradas; (O): Outputs ou saídas.

Fonte: Elaborada pelos autores.

Como pode ser observado na Tabela 4, nas DMUs ineficientes a taxa de mortalidade é a variável que merece mais atenção, sendo necessária uma redução de $87,73 \%$ para que todo o grupo passe a ser eficiente. Nesse sentido, esse resultado pode contribuir aos hospitais menos eficientes, já que a aplicação da DEA gerou padrões de eficiência que podem ser espelhados e pode orientar quanto às políticas de emprego dos recursos disponíveis no atendimento público de saúde por meio dos hospitais universitários. As demais variáveis da DEA não apresentaram potenciais de melhoria em valores relevantes.

Scherer et al. (2018) compararam hospitais universitários na Argélia, França e no Brasil. Os resultados do estudo apontaram que, em 2015, os serviços de emergência dos hospitais argelinos e franceses apresentaram uma taxa de ocupação superior a $100 \%$ na Argelia e de $85 \%$ na França. Nos hospitais brasileiros avaliados, HUB-UnB e HUPPEST-UFSC a taxa de ocupação em 2015 foi de 47,28\% e 75,85\%, respectivamente. No entanto, em relação à taxa de mortalidade, indicada nesse estudo como principal ponto de atenção para a gestão dos HUs brasileiros, a situação se inverte. Em 2015, a taxa de mortalidade do HUB-UnB foi de $6,46 \%$ e no HUPPEST-UFSC foi de 8 a $10 \%$. Nos outros dois países esse percentual foi bem menor: 2,80\% na Argélia e 0,4\% na França. A comparação do desempenho dos hospitais universitários nos três países reforça o cuidado que a gestão dos hospitais universitários brasileiros deve ter com este indicador.

Utilizando-se como parâmetro o indicador taxa de mortalidade, é possível conjecturar que a adesão ao modelo de gestão gerencial da EBSERH, por si só, não garante aos hospitais universitários brasileiros um melhor desempenho assistencial. Nos três países avaliados por Scherer et al. (2018), os hospitais universitários avaliados possuem uma estrutura colegiada na sua gestão, no entanto, no Brasil têm-se a figura da EBSERH, que não possui equivalente nos outros dois países. Scherer et al. (2018) apontam alguns problemas que podem explicar o desempenho inferior dos hospitais brasileiros. Segundo os autores, os dois hospitais brasileiros avaliados no estudo sofrem com absenteísmo e rotatividade. No HUPPEST-UFSC essa situação é agravada pelo grande volume de atendimento de pacientes graves num cenário de precariedade de estrutura e materiais (Scherer et al., 2018).

Na sequência, agruparam-se os hospitais universitários a partir da eficiência calculada por meio da DEA (Tabela 3). Para tanto, e com base na análise de clusters, foi possível identificar quatro grupos com diferentes níveis de 
eficiência: baixa, média baixa, média alta e alta. A Tabela 5 mostra a distribuição das DMUs por nível de eficiência.

\section{Tabela 5}

Grupos de DMUs por nível de eficiência

\begin{tabular}{|c|c|c|c|c|}
\hline $\begin{array}{r}\text { Faixa de } \\
\text { Eficiência }\end{array}$ & Nível & DMU & $\begin{array}{l}\text { Número } \\
\text { de DMUs }\end{array}$ & $\begin{array}{c}\text { Proporção } \\
(\%)\end{array}$ \\
\hline $0 \leq x \leq 36,85$ & $\begin{array}{l}\text { Eficiência } \\
\text { baixa }\end{array}$ & HUJBB-UFPA & 1 & 2,5 \\
\hline $\begin{array}{c}36,85<x \leq \\
66,49\end{array}$ & $\begin{array}{l}\text { Eficiência } \\
\text { média } \\
\text { baixa }\end{array}$ & $\begin{array}{l}\text { HUS-UFS, HUGV-UFAM, HUAP-UFF, HUGG- } \\
\text { UNIRIO, HUAC-UFCG, HUDMRCJ-FURG, } \\
\text { HUJM-UFMT, HUPAA-UFAL, HUJF-UFJF, HU- } \\
\text { UFPI, HE-UFPEL, MCO-UFBA, HUPES-UFBA }\end{array}$ & 13 & 32,5 \\
\hline $\begin{array}{c}66,49<x \leq \\
86,24\end{array}$ & $\begin{array}{l}\text { Eficiência } \\
\text { média } \\
\text { alta }\end{array}$ & $\begin{array}{l}\text { HULW-UFPB, HUWC-UFC, HUPHCP-UFSCAR, } \\
\text { HUB-UnB, HDT-UFT, HC-UFPE, HUOL-UFRN, } \\
\text { HUL-UFS, HUPPEST-UFSC }\end{array}$ & 9 & 22,5 \\
\hline $\begin{array}{c}86,24<x \leq \\
100\end{array}$ & $\begin{array}{l}\text { Eficiência } \\
\text { alta }\end{array}$ & $\begin{array}{l}\text { HU-UNIVASF, CHC-UFPR, HCU-UFU, HUAB- } \\
\text { UFRN, HUBFS-UFPA, HUJB-UFCG, HU-UFMA, } \\
\text { MEAC-UFC, MEJC-UFRN, MVFA-UFPR, HCU- } \\
\text { UFTM, HUMAP-UFMS, HC-UFMG, HU-UFGD, } \\
\text { HUSM-UFSM, HC-UFG, HUCAM-UFES }\end{array}$ & 17 & 42,5 \\
\hline
\end{tabular}

Fonte: Elaborada pelos autores.

A distribuição das DMUs por nível de eficiência (Tabela 5) evidencia uma maior concentração de hospitais com eficiência alta (17), seguindo-se o grupo de eficiência média baixa (13) e o de eficiência média alta (9). Apenas o HUJBBUFPA foi classificado como de eficiência baixa. A Tabela 6 mostra o resultado do teste de correlação entre os níveis de eficiência e as fontes de financiamento do custeio dos hospitais da amostra. Cabe destacar que o detalhamento do fluxo de receitas não é especificado pela EBSERH no painel de indicadores (fonte de coleta), o que constitui uma limitação da análise exposta na Tabela 6.

\section{Tabela 6}

Correlação entre os níveis de eficiência e o financiamento com receitas de fontes próprias e do SUS

\begin{tabular}{|c|c|c|}
\hline \multirow{2}{*}{ Darog } & \multicolumn{2}{|c|}{$\begin{array}{l}\text { Eficiência calculada por meio da } \\
\text { DEA }\end{array}$} \\
\hline & Correlação de Spearman & Sig \\
\hline $\begin{array}{l}\text { Porcentagem custeada com receitas de fontes próprias } \\
\text { e do sus }\end{array}$ & 0,180 & 0,266 \\
\hline
\end{tabular}

Fonte: Elaborada pelos autores.

Na Tabela 6, verifica-se que não há correlação estatisticamente significativa entre o nível de eficiência calculado por meio da DEA e a porcentagem de despesas custeada com fontes de receitas próprias e do SUS. Assim, não é possível inferir que uma maior participação de receitas próprias ou oriundas do SUS na matriz de custeio dos hospitais reflete-se em uma maior eficiência no atendimento assistencial. No mesmo sentido, ao explorar a relação entre o desempenho econômico-financeiro e o nível de eficiência no contexto 
hospitalar, Oliveira (2016) não identificou que os hospitais eficientes apresentavam melhores resultados econômico-financeiros.

A Tabela 7 mostra os resultados do teste não-paramétrico de MannWhitney, que indicam se há diferença estatisticamente significante entre os níveis de eficiência dos hospitais de diferentes portes (pequeno, médio, grande e especial).

\section{Tabela 7}

Teste de diferenças entre médias de Mann-Whitney

\begin{tabular}{|c|c|c|c|}
\hline Hipótese nula & Porte dos hospitais & Sig & Decisão \\
\hline \multirow{6}{*}{$\begin{array}{l}\text { O nível de } \\
\text { eficiência é igual } \\
\text { nos hospitais de } \\
\text { diferentes portes }\end{array}$} & $\begin{array}{l}\text { Pequeno porte e médio } \\
\text { porte }\end{array}$ & 0,273 & Não se rejeita a hipótese nula \\
\hline & $\begin{array}{l}\text { Pequeno porte e grande } \\
\text { porte }\end{array}$ & $0,023 *$ & Rejeita-se a hipótese nula \\
\hline & $\begin{array}{l}\text { Pequeno porte e porte } \\
\text { especial }\end{array}$ & 0,800 & Não se rejeita a hipótese nula \\
\hline & Médio porte e grande porte & 0,270 & Não se rejeita a hipótese nula \\
\hline & $\begin{array}{l}\text { Médio porte e porte } \\
\text { especial }\end{array}$ & 0,287 & Não se rejeita a hipótese nula \\
\hline & $\begin{array}{l}\text { Grande porte e porte } \\
\text { especial }\end{array}$ & $0,014^{*}$ & Rejeita-se a hipótese nula \\
\hline
\end{tabular}

Nota: $\left(^{*}\right)$ significância estatística de 5\%.

Fonte: Elaborada pelos autores.

Na Tabela 7, percebe-se que há diferenças estatisticamente significantes entre os níveis de eficiência calculados por meio da DEA, na comparação dos grupos de hospitais de pequeno porte e grande porte, bem como no grupo de hospitais de grande porte e porte especial, ao nível de significância de $5 \%$. Por outro lado, não há diferenças estatisticamente significantes entre os níveis de eficiência dos hospitais de pequeno porte e médio porte; pequeno porte e porte especial; médio porte e grande porte; e médio porte e porte especial.

Os dois únicos hospitais de pequeno porte (HUBFS-UFPA e HUJB-UFCG) apresentaram a maior média de eficiência, assinalando 100,0\%. Enquanto isso, 0 grupo dos 25 hospitais de grande porte apresentou a menor média de eficiência, de 74,35\%. Logo, embora os hospitais de maior porte aparentemente sejam capazes de um desenvolvimento mais avançado de controles gerenciais, como constatado por Espejo et al. (2015), isso não se refletiu em um índice de eficiência mais elevado. Também não foi confirmada a hipótese de Mendes (2011), de que o número ideal de leitos de um hospital universitário seria entre 100 e 450.

Apesar da aparente diminuição da eficiência dos hospitais em razão do aumento do porte, não é possível, com base no nível de eficiência calculado, inferir que o desempenho tende a cair com o crescimento do número de leitos, já que o grupo dos hospitais de porte especial, que são as maiores unidades em número de leitos (Ministério da Saúde, 1977), também apresentou um alto nível de eficiência. Dos três hospitais da amostra classificados como de porte especial, dois (CHC-UFPR e HCU-UFU) alcançaram a eficiência máxima (100\%). Esse resultado não corrobora a hipótese formulada por Mendes (2011) de que as deseconomias de escala vão se tornar importantes em hospitais com mais de 650 leitos. 


\section{CONCLUSÕES}

Dada a relevância dos hospitais universitários federais no contexto da saúde pública brasileira, não só pela missão de formar profissionais de saúde, mas também por exercer um relevante papel na assistência à saúde da população mais carente, o presente estudo analisou a eficiência do atendimento assistencial dos 40 hospitais universitários federais geridos pela EBSERH.

Os resultados revelaram que 10 dos 40 hospitais universitários analisados atingiram o índice de eficiência de 100\%. Verificou-se ainda que sete dos 10 hospitais que atingiram a fronteira de eficiência na dimensão assistência também foram classificados como eficientes pela pesquisa realizada por Martini et al. (2019), no que tange ao aspecto financeiro de diferentes DMUs por meio de distintas variáveis. Logo, os hospitais universitários classificados como eficientes na dimensão assistência e no aspecto financeiro podem ser considerados possíveis referências de bom desempenho, tomando-se por base os resultados das duas pesquisas. Essa análise foi possível pela aplicação do método DEA utilizado neste estudo, o qual gerou escores de eficiência para cada DMU, nesse caso, para cada hospital, e as DMUs mais eficientes ou com escore máximo passam a ser benchmarks às menos eficientes.

O estudo revelou que, dentre os hospitais que atingiram a fronteira de eficiência não há predominância de um porte específico, o mesmo ocorrendo com os hospitais que apresentaram os mais baixos índices de eficiência. Apesar de o maior porte dos hospitais resultar em um desenvolvimento mais avançado de controles gerenciais, como constatado por Espejo et al. (2015), isso não se refletiv em um índice de eficiência mais elevado.

Tomando-se por base o nível de eficiência médio dos hospitais de grande porte, também não foi corroborada a hipótese levantada por Mendes (2011), de que o número ideal de leitos por hospital situa-se entre 100 e 450, já que esse grupo apresentou o menor índice médio de eficiência. Outra afirmativa do autor que não se refletiu nos índices de eficiência dos hospitais universitários da amostra foi a de que existe uma deseconomia de escala para hospitais com mais de 650 leitos. Ademais, ao se confrontar a lista de hospitais classificados como eficientes em outras pesquisas, o HCU-UFU se destaca com o maior valor das despesas empenhadas. O citado hospital foi classificado também como eficiente por Lins et al. (2007), Lobo et al. (2009, 2016) e Martini et al. (2019).

A amostra do estudo, restrita aos 40 hospitais universitários federais geridos pela EBSERH, incluiu apenas dois hospitais do Estado do Rio de Janeiro. Não obstante a pequena representatividade, os dois hospitais fluminenses integram o grupo das cinco DMUs com os piores índices de eficiência.

Cabe destacar que, no que tange às melhorias a ser implementadas no grupo dos hospitais universitários federais visando a atingir a fronteira de eficiência, constatou-se que a taxa de mortalidade é a variável que merece mais atenção, sendo necessária uma redução de $87,73 \%$ no quadro atual para que todo o grupo da amostra se torne eficiente. Quanto a esse potencial de melhoria, convém destacar que Barreto (2015) afirma que o pay for performance (pagamento por desempenho) tem sido amplamente defendido como uma estratégia para implementar mudanças comportamentais em provedores de serviços de saúde, seja em nível individual ou coletivo. Logo, o pay for 
performance pode ser uma alternativa para melhoria do indicador taxa de mortalidade no âmbito dos hospitais universitários geridos pela EBSERH.

Não é possível inferir que a priorização do atendimento por meio do SUS ou a obtenção de receitas próprias reflete-se em maior eficiência no atendimento assistencial. Isso foi identificado a partir dos resultados do teste de correlação, que apontou não haver correlação estatisticamente significativa entre o nível de eficiência do atendimento assistencial dos hospitais e a porcentagem de despesas custeada com fontes de receitas próprias e do SUS.

A aplicação do teste de diferenças entre médias evidenciou a existência de diferenças significantes entre os níveis de eficiência, na comparação dos grupos de hospitais por porte, no caso dos hospitais de pequeno porte e grande porte. O grupo de hospitais de pequeno porte, por exemplo, atingiu a eficiência máxima (100\%). Também apresentou um bom desempenho o grupo de hospitais de porte especial, que apresentou diferenças significantes de eficiência, quando comparado ao grupo de hospitais de grande porte. O pior desempenho observado, considerando-se a média dos índices de eficiência, foi o do grupo de hospitais de grande porte.

Como limitações da pesquisa, menciona-se o fato de no momento da coleta não haver disponibilidade de dados completos no painel de indicadores da EBSERH para outros exercícios. No que tange à definição da amostra, não foi possível incluir hospitais universitários não vinculados à EBSERH, já que na principal fonte de dados, no caso o painel de indicadores, são divulgados dados apenas dos hospitais geridos pela EBSERH. Outra limitação do estudo é que não foram considerados, na eficiência calculada por meio da DEA, os procedimentos realizados, o tipo de especialização e o nível de complexidade dos hospitais universitários analisados.

Quanto à sugestão para pesquisas futuras, recomenda-se que sejam acrescentados ao modelo novos indicadores de desempenho, bem como outros hospitais universitários que não possuem contrato com a EBSERH. Outra sugestão para pesquisas futuras é que se procure identificar a relação existente entre o nível de eficiência dos hospitais universitários e o estágio de implantação do modelo de gestão da EBSERH, não investigado nesta pesquisa.

\section{REFERÊNCIAS}

Afonso, A., Schuknecht, L., \& Tanzi, V. (2010) Income distribution determinants and public spending efficiency. Journal of Economic Inequality, 8(3), 367-389. 10.1007/s10888-010-9138-z

Araújo, K. M., \& Leta, J. (2014). Os hospitais universitários federais e suas missões institucionais no passado e no presente. História, Ciências, Saúde-Manguinhos, 21(4), 1261-1281. https://doi.org/10.1590/S0104-59702014005000022

Avelar, A. B. A., Silva, A. S., Fouto, N. M. M. D. (2018). Evolução dos custos médicohospitalares de planos privados de assistência à saúde no Brasil. Revista de Gestão em Sistemas de Saúde, 7(1), 29-43. http://dx.doi.org/10.5585/rgss.v7i1.381 
Banker, R. D., Charnes, A., \& Cooper, W. W. (1984). Some models for estimating technical and scale inefficiencies in data envelopment analysis. Management Science, 30(9), 1078-1092. https://doi.org/10.1287/mnsc.30.9.1078

Barreto, J. O. M. (2015). Pagamento por desempenho em sistemas e serviços de saúde: uma revisão das melhores evidências disponíveis. Ciência \& Saúde Coletiva, 20, 1497-1514. https://doi.org/10.1590/1413-81232015205.01652014.

Bonacim, C. A. G., \& Araújo, A. M. P. (2010). Gestão de custos aplicada a hospitais universitários públicos: a experiência do Hospital das Clínicas da Faculdade de Medicina de Ribeirão Preto da USP. Revista de Administração Pública, 44(4), 903-931. https://doi.org/10.1590/S0034-76122010000400007

Borges, J. C. P., Barcelos, M., \& Rodrigues, M. S. (2018). Empresarização da saúde pública: o caso da EBSERH. Revista Pensamento Contemporâneo em Administração, 12(4), 75-90. https://doi.org/10.12712/rpca.v12i4.27119

Borgonovi, E., \& Compagni, A. (2013). Sustaining universal health coverage: the interaction of social, political, and economic sustainability. Valve in Health, 16(1), S34-S38. https://doi.org/10.1016/j.jval.2012.10.006

Cesconetto, A., Lapa, J. S., \& Calvo, M. C. M. (2008). Avaliação da eficiência produtiva de hospitais do SUS de Santa Catarina, Brasil. Cadernos de Saúde Pública, 24, 2407-2417. https://doi.org/10.1590/S0102-311X2008001000021

Charnes, A., Cooper, W. W., \& Rhodes, E. (1978). Measuring the efficiency of decision-making units. European Journal of Operational Research, 2(6), 429444. https://doi.org/10.1016/0377-2217(78)90138-8

Conselho Federal de Medicina. (2019). Hospitais universitários. Recuperado em 17 dezembro, 2019, de https://portal.cfm.org.br/index.php?option=com_content\&view=article\&id=2 8445:2019-09-23-13-00-34\&catid=33:hospitais-universitarios\&ltemid=46

Dantas, M. G. S., \& Boente, D. R. (2012). A utilização da análise envoltória de dados na medição de eficiência dos clubes brasileiros de futebol. Contabilidade Vista \& Revista, 23(2), 101-130. Recuperado de https://revistas.face.ufmg.br/index.php/contabilidadevistaerevista/article/vie $w / 1549$

Emenda Constitucional n. 19, de 04 de junho de 1998. (1998). Brasília. Recuperado em 17 dezembro, 2020, de http://www.planalto.gov.br/ccivil_03/Constituicao/Emendas/Emc/emc19.ht $\mathrm{m}$

Empresa Brasileira de Serviços Hospitalares. (n.d.a). Painéis de monitoramento. Recuperado em 19 dezembro, 2019, de http://ebserh.gov.br/paineis 
Empresa Brasileira de Serviços Hospitalares. (n.d.b). Sobre a rede EBSERH. Recuperado em 25 dezembro, 2019, de http://ebserh.gov.br/sobre-a-redeebserh

Empresa Brasileira de Serviços Hospitalares. (n.d.c). Sobre os hospitais universitários. Recuperado em 17 dezembro, 2018, de http://ebserh.gov.br/hospitaisuniversitarios-federais

Espejo, M. M. S. B., Portulhak, H., \& Martins, D. B. (2015). Práticas de controle gerencial em hospitais universitários federais. Gestão \& Regionalidade, 31(92), 39-52. https://doi.org/10.13037/gr.vol31n92.2421

Farrell, M. J. (1957). The measurement of productive efficiency. Journal of the Royal Statistical Society: Series A (General), 120(3), 253-281. https://doi.org/10.2307/2343100

Fávero, L. P. L., Belfiore, P. P., Silva, F. L., \& Chan, B. L. (2009). Análise de dados: Modelagem multivariada para tomada de decisões. Rio de Janeiro: Elsevier.

Hadad, S., Hadad, Y., \& Simon-Tuval, T. (2013). Determinants of healthcare system's efficiency in OECD countries. The European Journal of Health Economics, 14(2), 253-265.

Hair, J. F., Júnior, Black, W. C., Babin, B. J. Anderson, R. E., \& Tatham, R. L. (2009). Análise multivariada de dados (5a ed.). Porto Alegre: Bookman.

Kirigia, J. M., Emrouznejad, A., Sambo, L. G., Munguti, N., \& Liambila, W. (2004). Using data envelopment analysis to measure the technical efficiency of public health centers in Kenya. Journal of Medical Systems, 28(2), 155-166. 10.1023/b:joms.0000023298.31972.c9

Kohl, S., Schoenfelder, J., Fügener, A., \& Brunner, J. O. (2019). The use of data envelopment analysis (DEA) in healthcare with a focus on hospitals. Health Care Management Science, 22(2), 245-286. https://doi.org/10.1007/s10729$\underline{018-9436-8}$

Lei n. 12.550, de 15 de dezembro de 2011. Autoriza o Poder Executivo a criar a empresa pública denominada Empresa Brasileira de Serviços Hospitalares EBSERH; acrescenta dispositivos ao Decreto-Lei $n^{\circ} 2.848$, de 7 de dezembro de 1940 - Código Penal; e dá outras providências. Brasília, 2011. Recuperado em 17 dezembro, 2020, de http://www.planalto.gov.br/CCIVIL_03/_Ato20112014/2011/Lei/L12550.htm

Lee, S., \& Kim, C. (2018). Estimation of association between healthcare system efficiency and policy factors for public health. Applied Sciences, 8(12), 2674. https://doi.org/10.3390/app8122674

Lins, M. E., Lobo, M. S. C., Silva, A. C. M., Fiszman, R., \& Ribeiro, V. J. P. (2007). O uso da análise envoltória de dados (DEA) para avaliação de hospitais 
universitários brasileiros. Ciência \& Saúde Coletiva, 12, 985-998. https://doi.org/10.1590/S1413-81232007000400020

Lobo, M. S. C., Silva, A. C. M., Lins, M. P. E., \& Fiszman, R. (2010). Avaliação de desempenho e integração docente-assistencial nos hospitais universitários. Revista de Saúde Pública, 44, 581-590. https://doi.org/10.1590/S0034$\underline{89102010000400001}$

Lobo, M. S. C., Silva, A. C. M., Lins, M. P. E., \& Fiszman, R. (2009). Impacto da reforma de financiamento de hospitais de ensino no Brasil. Revista de Saúde Pública, 43, 437-445. https://doi.org/10.1590/S0034-89102009005000023

Lobo, M. S. C., Silva, A. C. M., Lins, M. P. E., \& Fiszman, R. (2011). Influência de fatores ambientais na eficiência de hospitais de ensino. Epidemiologia $e$ Serviços de Saúde, 20(1), 37-45. http://dx.doi.org/10.5123/S1679$\underline{49742011000100005}$

Lobo, M. S. C., Rodrigues, H. C., André, E. C. G. Azeredo, J. A., \& Lins, M. P. E. (2016). Análise envoltória de dados dinâmica em redes na avaliação de hospitais universitários. Revista de Saúde Pública, 50, 22. https://doi.org/10.1590/S1518$\underline{8787.2016050006022}$

Martini, B. P., Machado, D. G., Menezes, G., \& Souza, M. A. (2019). Avaliação financeira de eficiência relativa: uma análise em hospitais universitários federais do Brasil. Anais do Congresso Anpcont, São Paulo, SP, Brasil, 13.

Mello, J. C. C. B. S., Meza, L. A., Gomes, E. G., \& Biondi, L., Neto. (2005). Curso de análise de envoltória de dados. Simpósio Brasileiro de Pesquisa Operacional, 37, 2520-2547.

Mendes, E. V. As redes de atenção à saúde. (2011). Brasília: Organização PanAmericana da Saúde. Recuperado em 01 fevereiro, 2020, de http://www.paho.org/bra/index.php?option=com_docman\&view=downloa d\&category_slug=servicos-saude-095\&alias=1402-as-redes-atencao-a-saude2a-edicao-2\&ltemid=965

Ministério da Saúde. (1977). Conceitos e definições. Brasília. Recuperado em 31 janeiro, 2020,

http://bvsms.saude.gov.br/bvs/publicacoes/0117conceitos.pdf

Ministério da Saúde. (n.d.). Departamento de informática do SUS (DATASUS). Recuperado em 25 dezembro, 2019, de http://datasus.saude.gov.br

Miranda, G. J., Carvalho, C. E., Martins, V. F., \& Faria, A. F. (2007). Custeio ABC no ambiente hospitalar: um estudo nos hospitais universitários e de ensino brasileiros. Revista Contabilidade \& Finanças, 18(44), 33-43. https://doi.org/10.1590/S1519-70772007000200004

Nascimento, J. C. H. B., Nossa, V., Bernardes, J. R., \& Sousa, W. D. (2015). A eficiência dos maiores clubes de futebol brasileiros: evidências de uma análise 
longitudinal no período de 2006 a 2011 . Contabilidade Vista \& Revista, 26(2), 137-161.

https://revistas.face.ufmg.br/index.php/contabilidadevistaerevista/article/vie w/2707

Oliveira, H. C. S. (2016). Desempenho do contrato de gestão e a situação econômico-financeira dos hospitais gerais administrados organizações sociais de saúde: uma análise no estado de São Paulo. Dissertação de Mestrado, Universidade de São Paulo, São Paulo, SP, Brasil.

Peixoto, M. G. M. (2016). Análise envoltória de dados e análise de componentes principais: uma proposta de medição do desempenho de organizações hospitalares sob a perspectiva de hospitais universitários federais do Brasil. Tese de Doutorado, Universidade de São Paulo, São Paulo, SP, Brasil.

Peña, C. R. (2008). Um modelo de avaliação da eficiência da administração pública através do método análise envoltória de dados (DEA). Revista de Administração Contemporânea, 12(1), 83-106. https://doi.org/10.1590/S1415$\underline{65552008000100005}$

Scherer, M. D. D. A., Conill, E. M., Jean, R., Taleb, A., Gelbcke, F. L., Pires, D. E. P. D., \& Joazeiro, E. M. G. (2018). Desafios para o trabalho em saúde: um estudo comparado de Hospitais Universitários na Argélia, Brasil e França. Ciência \& Saúde Coletiva, 23, 2265-2276. https://doi.org/10.1590/1413$\underline{81232018237.08762018}$

Silva, B. N., Costa, M. A. S., Abbas, K., \& Galdamez, E. V. C. (2017). Eficiência hospitalar das regiões brasileiras: um estudo por meio da análise envoltória de dados. Revista de Gestão em Sistemas de Saúde, 6(1), 76-91. http://dx.doi.org/10.5585/rgss.v6il.314

Silva, M. Z., Moretti, B. R., \& Schuster, H. A. (2016). Avaliação da eficiência hospitalar por meio da análise envoltória de dados. Revista de Gestão em Sistemas de Saúde, 5(2), 100-114. http://dx.doi.org/10.5585/rgss.v5i2.248

Sousa, M. C. S., \& Ramos, F. S. (1999). Eficiência técnica e retornos de escala na produção de serviços públicos municipais: o caso do Nordeste e do Sudeste brasileiros. Revista Brasileira de Economia, 53(4), 433-461. https://doi.org/10.1590/\$0034-71401999000400002

Souza, I. V., Nishijima, M., \& Rocha, F. (2010). Eficiência do setor hospitalar nos municípios paulistas. Economia Aplicada, 14(1), 51-66. https://doi.org/10.1590/S1413-80502010000100004

Tesouro Nacional. (2018). Aspectos fiscais da saúde no Brasil. Recuperado 15 dezembro, 2019 ,

http://www.tesourotransparente.gov.br/publicacoes/aspectos-fiscais-dasaude-no-brasil/2018/30 
Tonelotto, D. P., Crozatti, J., Moraes, V. M., \& Righetto, P. (2019). Hospitais de alta complexidade do Estado de São Paulo: uma análise comparativa dos níveis de eficiência obtidos pelos modelos de gestão de administração direta e de organização social. Administração Pública e Gestão Social, 11(4), 59-77. https://doi.org/10.21118/apgs.v4i11.7175

Venturini, L. D. B., Souza, R. L., \& Bianchi, M. (2020). Eficiência na alocação dos recursos públicos do Poder Judiciário: um estudo na $4^{\mathrm{a}}$ Região Federal. Contabilidade Vista \& Revista, 31(3), 26-51. https://doi.org/10.22561/cvr.v31i2.5178

Xu, K., Soucat, A., Kutzin, J., Brindley, C., Maele, N. V., Touré, H., Garcia, M. A., Li, D., Barroy, H., Flores, G., Roubal, T., Indikadahena, C., Cherilova, V., \& Siroka, A. (2018). Public spending on health: a closer look at global trends. World Health Organization. Retrieved December 15, 2020, from https://apps.who.int/iris/bitstream/handle/10665/276728/WHO-HIS-HGF-HFWorkingPaper-18.3-eng.pdf

\section{CONTRIBUIÇÕES DOS AUTORES}

\begin{tabular}{|l|c|c|c|}
\hline \multicolumn{1}{|c|}{ Contribuição } & $\begin{array}{c}\text { Samuel } \\
\text { Cavalcante } \\
\text { Mota }\end{array}$ & $\begin{array}{c}\text { Antonio } \\
\text { Rafael Valério } \\
\text { de Oliveira }\end{array}$ & $\begin{array}{c}\text { Alessandra } \\
\text { Carvalho de } \\
\text { Vasconcelos }\end{array}$ \\
\hline $\begin{array}{l}\text { 1. Idealização e concepção do assunto e } \\
\text { tema da pesquisa }\end{array}$ & $\checkmark$ & $\checkmark$ & $\checkmark$ \\
\hline 2. Definição do problema de pesquisa & $\checkmark$ & $\checkmark$ & $\checkmark$ \\
\hline 3. Desenvolvimento da Plataforma Teórica & $\checkmark$ & $\checkmark$ & \\
\hline $\begin{array}{l}\text { 4. Delineamento da abordagem } \\
\text { metodológica da pesquisa }\end{array}$ & $\checkmark$ & $\checkmark$ & $\checkmark$ \\
\hline 5. Coleta de dados & $\checkmark$ & $\checkmark$ & \\
\hline $\begin{array}{l}\text { 6. Análises e interpretações dos dados } \\
\text { coletados }\end{array}$ & $\checkmark$ & $\checkmark$ & \\
\hline 7. Conclusões da pesquisa & $\checkmark$ & $\checkmark$ & \\
\hline 8. Revisão crítica do manuscrito & & & $\checkmark$ \\
\hline $\begin{array}{l}\text { 9. Redação final do manuscrito, conforme as } \\
\text { normas estabelecidas pela Revista. }\end{array}$ & $\checkmark$ & $\checkmark$ & $\checkmark$ \\
\hline 10. Orientação & & & $\checkmark$ \\
\hline
\end{tabular}

